07;09

\title{
Температурные зависимости оптических свойств наночастиц алюминия
}

\author{
(C) А.В. Каленский ${ }^{1}$, А.А. Звеков ${ }^{2}$ \\ ${ }^{1}$ Кемеровский государственный университет, Кемерово \\ ${ }^{2}$ Федеральный исследовательский центр угля и углехимии СО РАН, \\ Кемерово \\ E-mail: kriger@kemsu.ru.
}

Поступило в Редакцию 27 декабря 2016 г.

Проведен анализ спектральных зависимостей оптических свойств алюминия при различных температурах. Предложены и апробированы аппроксимирующие выражения для мнимой части диэлектрической проницаемости. Выполнен расчет коэффициента отражения при нормальном падении света и оптических свойств наночастиц с радиусом $150 \mathrm{~nm}$ в температурном интервале 298-933 K для практически важных длин волн. Показано, что для алюминия наблюдается нетипичное поведение оптических свойств: повышение коэффициента отражения и уменьшение коэффициента эффективности поглощения наночастиц при росте температуры, что связано с вкладом межзонных переходов в оптические свойства алюминия.

DOI: 10.21883/PJTF.2017.11.44699.16642

Исследование влияния температуры на оптические свойства металлов и состоящих из них наночастиц является важной практической задачей. При получении наночастиц методом абляции металлов [1] температурная зависимость коэффициента отражения приводит к коррекции значения критической плотности энергии импульса [2]. Изменение оптических свойств пленки молибдена при лазерном воздействии вызывает уменьшение зоны абляции [3,4]. В [5] показано, что рост температуры приводит к повышению коэффициента эффективности поглощения наночастиц серебра на длине волны основной гармоники неодимового лазера, что может вызывать нелинейные оптические эффекты в поле лазерного излучения. Данные эффекты необходимо учитывать при структурировании материалов лазерным излучением [6]. В то же время экспериментальное исследование данных зависимостей представляет собой нетривиальную задачу и для большинства металлов 
имеются только отрывочные сведения, которых недостаточно для непосредственного прогнозирования оптических свойств наночастиц при различных температурах. Цель работы - получить аппроксимирующие выражения для диэлектрической проницаемости алюминия при различных температурах, рассчитать температурные зависимости коэффициентов отражения металла и эффективности поглощения наночастиц алюминия.

Результаты измерения поглощения света напыленными пленками алюминия в вакууме при температурах 198, 298, 404 и 552 К, выполненные в области спектра от 0.64 до $2.5 \mathrm{eV}$ в [7], приведены на рис. $1, a$ (кривые $1,3,5$ и 7). На спектральной зависимости мнимой части диэлектрической проницаемости $\varepsilon_{2}(E)$ проявляется полоса с локальным максимумом в области $1.5 \mathrm{eV}$, форма которой отличается от лоренцевской [7], связанная с межзонным поглощением [8,9]. В [2,10] предложено использовать модель критических точек для описания оптических констант алюминия, но аппроксимация выполнена только в видимой области без анализа влияния температуры [10]. Возрастание $\varepsilon_{2}$ при уменьшении $E$ в области менее $1 \mathrm{eV}$ связано не только с внутризонным поглощением света, но и со слабой полосой межзонного поглощения с максимумом в районе $0.5 \mathrm{eV}$. Нами использовано следующее выражение для аппроксимации зависимости $\varepsilon_{2}(E)$ в области $0.64-2.5 \mathrm{eV}$ :

$$
\varepsilon_{2}(E)=A_{1} E^{A_{2}}+A_{3} E^{A_{4}}\left[1+\exp \left(-A_{5}\left(E-A_{6}\right)\right)\right]
$$

где энергия кванта выражена в $\mathrm{eV}$. Предложенный вид зависимости $\varepsilon_{2}(E)$ определяется изменением $\varepsilon_{2}$ в широком интервале с несколькими локальными экстремумами при относительно небольшом изменении аргумента. Параметры уравнения (1) вычислялись для каждой из 4 температур. После этого температурная зависимость параметров $A$ (для $A_{3}$ и $A_{5}$ - обратных величин $A$ ) аппроксимировалась квадратным трехчленом. В результате получены следующие выражения для температурных зависимостей параметров:

$$
\begin{gathered}
A_{1}=25.6829+5.9058 \cdot T+1.1469 \cdot T^{2}, \\
A_{2}=1.2711-0.9069 \cdot T+0.166 \cdot T^{2}, \\
A_{3}=\left(-2.2597+5.5674 \cdot 10^{-3} \cdot T-1.0550 \cdot 10^{-3} \cdot T^{2}\right)^{-1}, \\
A_{4}=-9.2965+5.9985 \cdot T-1.9412 \cdot T^{2},
\end{gathered}
$$

Письма в ЖТФ, 2017, том 43, вып. 11 


$$
\begin{gathered}
A_{5}=\left(3.3477 \cdot 10^{-2}+3.4032 \cdot 10^{-2} \cdot T+2.9111 \cdot 10^{-3} \cdot T^{2}\right)^{-1}, \\
A_{6}=1.7026-0.2834 \cdot T+5.6784 \cdot 10^{-2} \cdot T^{2},
\end{gathered}
$$

где температура нормирована на $300 \mathrm{~K}$. Результаты аппроксимации с помощью выражений (1) и (2) также приведены на рис. 1, a (кривые $2,4,6,8)$. Таким образом, эвристическое выражение (1) позволяет получить описание экспериментальных зависимостей с точностью, достаточной для прогнозирования оптических постоянных алюминия при промежуточных температурах. Максимальное отклонение рассчитанных значений от литературных составляет 14\% (коэффициент корреляции $>0.99)$. В области энергий кванта более $1.1 \mathrm{eV}$ максимальное отклонение не превышает 10\%. Теоретические зависимости, основанные на методе псевдопотенциала $[8,9]$, при воспроизведении характерных особенностей экспериментальных результатов приводят к недостаточной точности их описания, особенно при повышенных температурах, где отклонение составляет $20 \%$, являясь систематическим.

Для расчета действительной части диэлектрической проницаемости использовались соотношения Крамерса-Кронига. При этом для функции $\varepsilon_{2}(E)$ использовалось выражение (1) с параметрами (2) при энергии кванта $E \geqslant 0.45 \mathrm{eV}$. В области $E<0.45 \mathrm{eV}$ использовалось выражение, следующее из теории Друде:

$$
\varepsilon_{1 D}(E)=1-\frac{E_{p}^{2}}{\left(E^{2}+\Gamma^{2}\right)}, \quad \varepsilon_{2 D}(E)=\frac{\Gamma E_{p}^{2}}{E\left(E^{2}+\Gamma^{2}\right)},
$$

где $E_{p}=\hbar \sqrt{4 \pi n_{e} e^{2} / m_{e}}-$ энергия плазменных колебаний, $\Gamma=$ $=n_{e} e^{2}\left(m_{e f f} m_{e} \sigma_{0}\right)^{-1}-$ релаксационный параметр, выраженный в единицах энергии, $e-$ заряд электрона, $m_{e}-$ масса покоя электрона, $n_{e}-$ объемная концентрация валентных электронов, $m_{e f f}-$ отношение эффективной массы электрона в металле к массе покоя электрона, $\sigma_{0}$ статическая проводимость.

Результаты расчетов $\varepsilon_{1}(E)$ с использованием выражений (1)-(3) приведены на рис. 1, $b$ по модулю при температурах 198 (1), 298 (2), 404 (3) и $552 \mathrm{~K}$ (4). Одновременно отображена справочная зависимость при комнатной температуре (5) [11]; выделяется локальный минимум зависимости $\varepsilon_{1}(E)$ при энергии кванта около $1.5 \mathrm{eV}$, соответствующий полосе межзонного поглощения. Как и на зависимости $\varepsilon_{2}(E)$, интенсивность полосы уменьшается с ростом температуры.

Письма в ЖТФ, 2017, том 43, вып. 11 

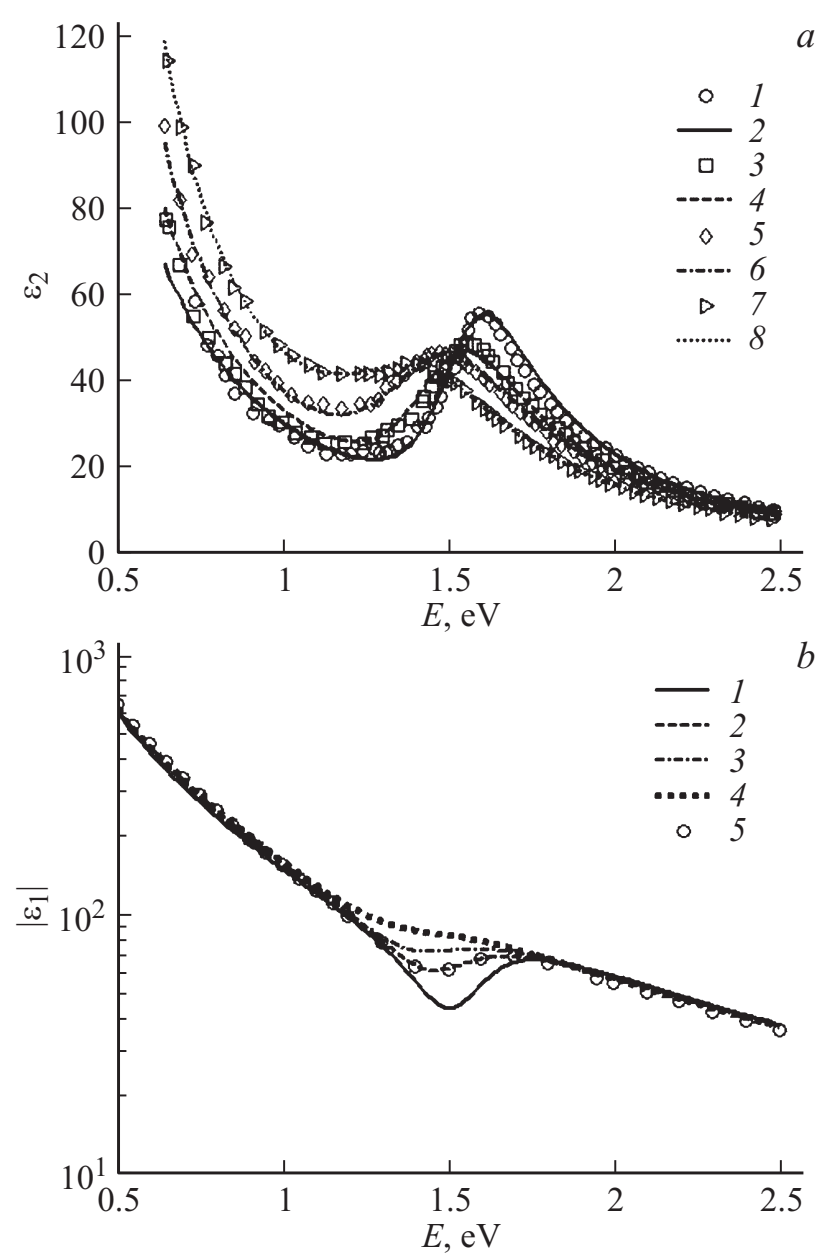

Рис. 1. Спектральные зависимости диэлектрической проницаемости алюминия: $a$ - мнимая часть при температурах 198 (1 и 2), 298 (3 и 4), 404 (5 и 6) и $552 \mathrm{~K}$ (7 и 8$)$, измеренная в работе [7] $(1,3,5,7)$ и аппроксимированная выражениями (1)-(2) $(2,4,6,8) ; b$ - действительная часть, рассчитанная по соотношениям Крамерса-Кронига при температурах 198 (1), 298 (2), 404 (3) и $552 \mathrm{~K}(4)$, и справочная зависимость при температуре $298 \mathrm{~K}$ [11] (5).

Письма в ЖТФ, 2017, том 43, вып. 11 

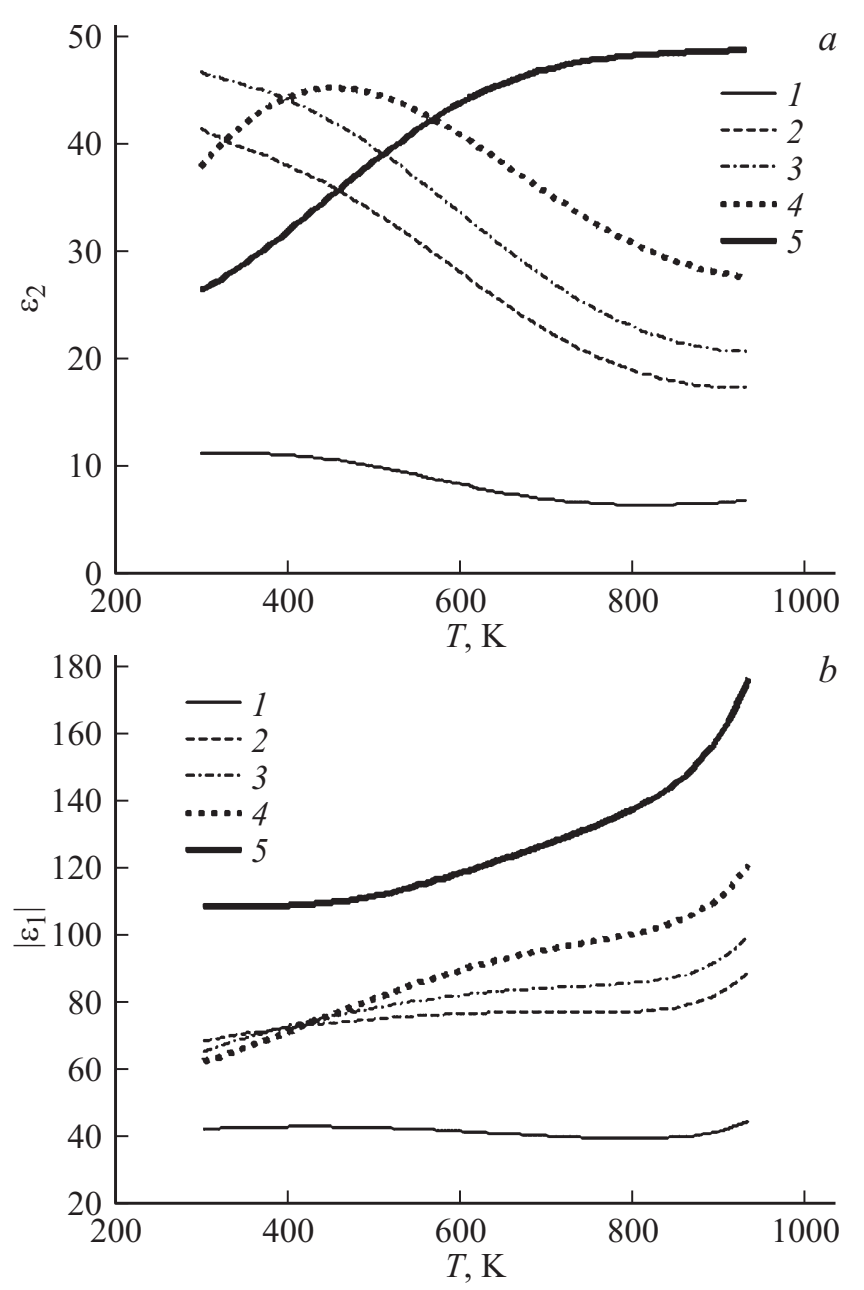

Рис. 2. Рассчитанные температурные зависимости мнимой $(a)$ и действительной $(b)$ части диэлектрической проницаемости алюминия и френелевского коэффициента отражения света при нормальном падении (c) при энергии кванта 2.33 (1), $1.66(2), 1.56$ (3), 1.42 (4) и $1.17 \mathrm{eV}(5)$. 


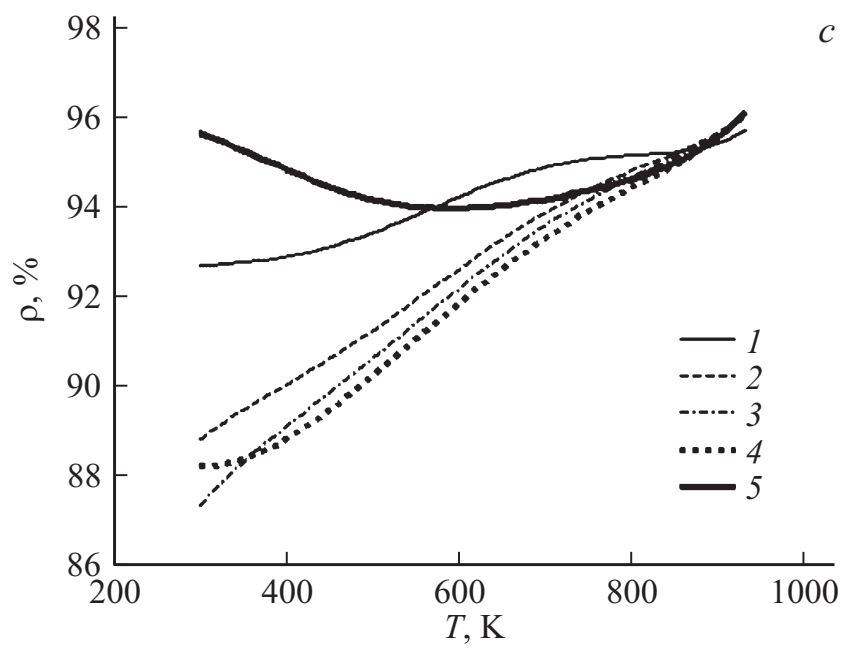

Рис. 2 (продолжение).

На рис. 2, $a$ и $b$ представлены результаты расчетов мнимой $\varepsilon_{2}(T)$ и действительной $\varepsilon_{1}(T)$ частей диэлектрической проницаемости алюминия по выражениям (1)-(3) при энергиях кванта электромагнитного излучения 2.33 (1), 1.66 (2), 1.56 (3), 1.42 (4) и $1.17 \mathrm{eV}$ (5). Выбор энергий кванта определяется рабочими частотами современных лазеров, положением вершины и краев межзонной полосы поглощения. Действительная часть диэлектрической проницаемости при повышении температуры имеет тенденцию к уменьшению (модуль растет) при $E$ менее $2 \mathrm{eV}$. Наибольшее уменьшение в диапазоне $298-933 \mathrm{~K}$ наблюдается в случае первой гармоники неодимового лазера. По мере увеличения энергии кванта разность между значениями $\varepsilon_{1}$ в точке плавления и при комнатной температуре уменьшается, составляя 20.5 при $E=1.66 \mathrm{eV}$. В случае второй гармоники неодимового лазера появляется небольшой локальный максимум при температуре $800 \mathrm{~K}$ с амплитудой -39.4 , тогда как при комнатной температуре величина $\varepsilon_{1}$ составляет -42.4 .

Увеличение температуры приводит к росту мнимой части диэлектрической проницаемости для энергии кванта $1.17 \mathrm{eV}$ и ее уменьшению при $E=1.56 \mathrm{eV}$ и более. На зависимости $\varepsilon_{2}(T)$, рассчитанной при

Письма в ЖТФ, 2017, том 43, вып. 11 
$E=1.42 \mathrm{eV}$, наблюдается локальный максимум при температуре $450 \mathrm{~K}$ с амплитудой 45.1. Причины появления максимума следуют из рис. 1. При увеличении температуры происходит батохромный сдвиг полосы межзонного поглощения алюминия с одновременным падением ее интенсивности. Данный сдвиг вначале приводит к росту величины $\varepsilon_{2}$ при энергии кванта $1.42 \mathrm{eV}$, затем дальнейшее смещение полосы с уменьшением ее интенсивности проявляется как уменьшение $\varepsilon_{2}$ до значения 27.5 в точке плавления.

На рис. 2,c представлены рассчитанные зависимости коэффициента френелевского отражения алюминия при нормальном падении света $\rho$ от температуры. Во всех случаях, кроме энергии кванта $1.17 \mathrm{eV}$, наблюдается увеличение коэффициента отражения. Разница между значениями $\rho$ при различных $E$ уменьшается по мере повышения температуры так, что $\rho$ стремится к величине $\sim 96 \%$ в точке плавления. Для основной гармоники неодимового лазера на зависимости $\rho(T)$ наблюдается минимум при температуре $590 \mathrm{~K}$.

Расчеты оптических свойств наночастиц алюминия выполнены в рамках теории Ми с учетом объемного расширения металла для наночастиц с радиусом $150 \mathrm{~nm}$ при комнатной температуре в вакууме по методике, описанной в $[12,13]$ и адаптированной в [5]. Рост температуры от 298 до $933 \mathrm{~K}$ приводит к уменьшению коэффициента эффективности рассеяния от 1.997 до 1.887 при энергии кванта $1.17 \mathrm{eV}$. Для остальных использованных значений энергии кванта более выражена тенденция к возрастанию $Q_{s c a}$ в пределах $5 \%$. Данный результат на качественном уровне согласуется с [5], где было выявлено слабое влияние температуры на коэффициент эффективности рассеяния света наночастицами серебра на длине волны $1060 \mathrm{~nm}$. Рассчитанные температурные зависимости коэффициента эффективности поглощения наночастицы алюминия радиусом $150 \mathrm{~nm}$ приведены на рис. 3. В случае энергии кванта $1.17 \mathrm{eV}$ видно формирование максимума $Q_{a b s}$ при $580 \mathrm{~K}$, амплитуда которого составляет 0.164, превышая на $37.2 \%$ значение при комнатной температуре и на 59.3\% при температуре плавления. При уменьшении радиуса наночастицы максимум сдвигается в область больших температур, наблюдаясь, например, при $620 \mathrm{~K}$ в случае радиуса наночастиц $50 \mathrm{~nm}$. Для всех остальных значений энергии кванта при повышении температуры от комнатной до точки плавления наблюдается тенденция к уменьшению $Q_{a b s}$ по мере роста температуры, более выраженная при уменьшении $E$. Наблюдаемые закономерности отли-

Письма в ЖТФ, 2017, том 43, вып. 11 


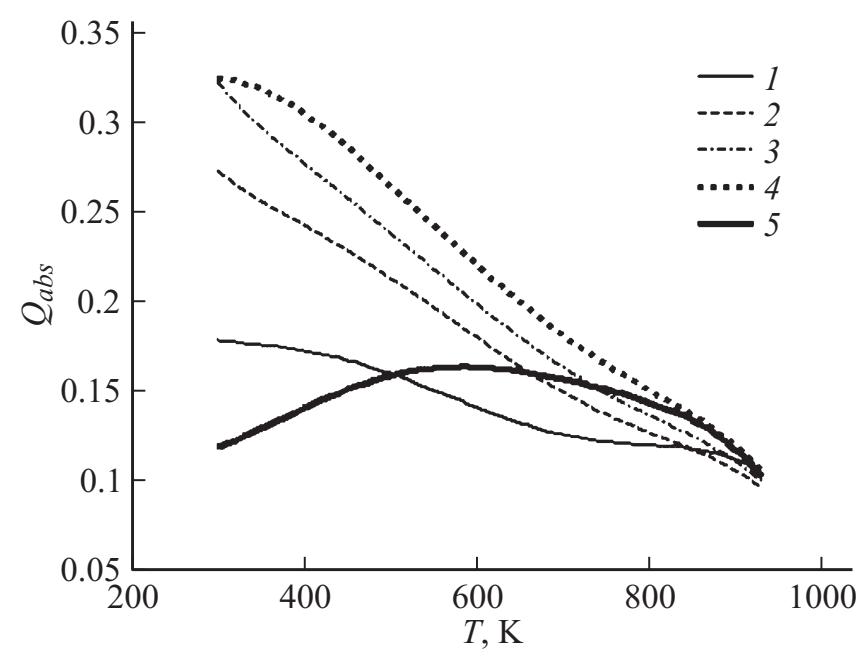

Рис. 3. Рассчитанные температурные зависимости коэффициентов эффективности поглощения света с энергией кванта 2.33 (1), 1.66 (2), 1.56 (3), 1.42 (4) и $1.17 \mathrm{eV}(5)$ наночастицами алюминия с радиусом $150 \mathrm{~nm}$.

чаются от [5], где наблюдалось близкое к линейному увеличение $Q_{a b s}$ наночастиц серебра при росте температуры.

Необычный характер результатов расчетов заключается в том, что увеличение температуры приводит к уменьшению коэффициента эффективности поглощения. Данное поведение является аномальным для металлов, поскольку рост температуры типично приводит к увеличению скорости диссипации энергии. Это должно приводить к уменьшению $Q_{s c a}$ и росту $Q_{a b s}[5]$. В области $E$ порядка $1.5 \mathrm{eV}$ основной вклад в оптические свойства алюминия вносит температурно-чувствительная полоса межзонного поглощения. Благодаря этому с ростом температуры $\varepsilon_{2}$ уменьшается при всех рассмотренных энергиях кванта, кроме $1.17 \mathrm{eV}$. При $E=1.17 \mathrm{eV}$ наблюдается возрастание $\varepsilon_{2}$, но не настолько выраженное, как по теории Друде. Это приводит к участку возрастания $Q_{a b s}$ до температуры порядка $600 \mathrm{~K}$, после чего начинается его уменьшение. С температурными зависимостями коэффициентов эффективности поглощения (рис. 3) коррелирует поведение коэффициента отражения $\rho(T)$ (рис. 2,c).

Письма в ЖТФ, 2017, том 43, вып. 11 
Таким образом, проанализированы оптические свойства алюминия и получены аппроксимирующие выражения для мнимой части диэлектрической проницаемости. Показано, что коэффициент отражения металла и коэффициент эффективности поглощения наночастиц демонстрирует аномальную температурную зависимость.

Работа выполнена при финансовой поддержке Министерства образования и науки РФ (НИР № 3.5363.2017/8.9).

\section{Список литературы}

[1] Старинский С.В., Шухов Ю.Г., Булгаков А.В. // Письма в ЖТФ. 2016. Т. 42. B. 8. C. 45.

[2] Marla D., Bhandarkar U.V., Joshi S.S. // J. Phys. D. 2014. V. 47. N 10. P. 105306.

[3] Казанский Н.Л., Полетаев С.Д. // ЖТФ. 2016. Т. 86. В. 9. С. 1.

[4] Волков А.В., Казанский Н.Л., Моисеев О.Ю. и др. // ЖТФ. 2016. Т. 86. В. 4. C. 101.

[5] Каленский А.В., Звеков А.А., Никитин А.П. // ЖПС. 2016. Т. 83. В. 6. С. 972.

[6] Гачкевич Е.И., Ивлев Г.Д., Малевич В.Л. // Письма в ЖТФ. 2015. Т. 41. В. 1. C. 43.

[7] Mathewson A.G., Myers H.P. // J. Phys. F. 1972. V. 2. N 2. P. 403.

[8] Ashcroft N.W., Sturm K. // Phys. Rev. B. 1971. V. 3. N 6. P. 1898.

[9] Brust D. // Phys. Rev. B. 1970. V. 2. N 4. P. 818.

[10] Barchiesi D., Grosges Th. // J. Nanophotonics. 2014. V. 8. N 1. P. 083097.

[11] Palik E.D. Handbook of Optical Constants. San Diego: Academic Press Inc., 1985. V. 1. P. 405.

[12] Ambrosio L.A. // J. Quant. Spectr. Rad. Transfer. 2016. V. 180. P. 147.

[13] Kolwas K., Derkachova A., Shopa M. // J. Quant. Spectr. Rad. Transfer. 2009. V. 110. N 14-16. P. 1490. 